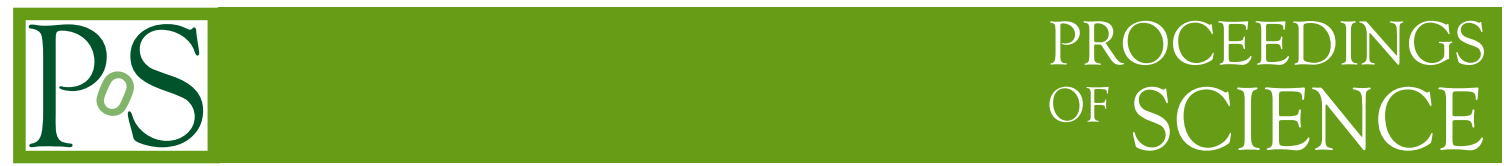

\title{
The $K^{+} \rightarrow \pi^{+} v \bar{v}$ experiment at CERN
}

\section{Giuseppe Ruggiero*}

Scuola Normale Superiore and INFN, Pisa, Italy

E-mail: giuseppe.ruggiero@cern.ch

The P326 proposal for an experiment to measure the branching ratio of the very rare kaon decay $K^{+} \rightarrow \pi^{+} v \bar{v}$ at the CERN SPS is described. The proposed experiment aims to collect about 80 $K^{+} \rightarrow \pi^{+} v \bar{v}$ events with a $10 \%$ of background in two years of data taking. The status of the project, the $R \& D$ and the future perspectives for the experiment are discussed.

KAON International Conference

May 21-25 2007

Laboratori Nazionali di Frascati dell'INFN, Rome, Italy

*Speaker. 


\section{Introduction}

The $K^{+} \rightarrow \pi^{+} v \bar{v}$ decay is a flavor changing neutral current process which proceeds through box and purely electroweak penguin diagrams. The short distance contributions largely dominate in the matrix element, while c-quark contributions have been evaluated at NNLO order giving an uncertainty of about 5\% [1]. This is the only source of theoretical error because the hadronic matrix element can be parametrized in terms of the branching ratio of the $K^{+} \rightarrow \pi^{0} e^{+} v$ decay, which is well known experimentally [2]. The computed value is $(8.0 \pm 1.1) \times 10^{-11}$, where the error is dominated by the uncertainty in the knowledge of the CKM matrix elements. Such extreme theoretical clarity, unique in $K$ and $B$ physics, makes this decay, together with the $K_{L} \rightarrow \pi^{0} v \bar{v}$ decay, extremely sensitive to new physics contributions both in Minimal Flavour Violation (MFV) and non-MFV scenarios $[3,4,5]$.

Up to now $3 K^{+} \rightarrow \pi^{+} v \bar{v}$ events have been observed [6], but a $10 \%$ accuracy measurement of the branchig ratio is required to provide a significative test of new physics scenarios. This is the goal of the proposed NA48/3 experiment at CERN-SPS [7]. It aims to collect about $80 K^{+} \rightarrow \pi^{+} v \bar{v}$ events keeping the background contamination at the level of $10 \%$.

\section{The P-326 proposal}

The NA48/3 experiment will be based on the NA48 apparatus at CERN and will make use of the same CERN-SPS beam line which produced the kaon beam for the NA48 experiment. The R\&D program for this experiment, started in 2006, is continuing in 2007. The data taking should start in 2010.

The layout of the experiment is shown in figure 1. The goal of the experiment can be achieved by exploiting a decay in flight technique which allows $10 \%$ signal acceptance and by using a beam line able to provide of the order of $10^{13}$ kaon decays.

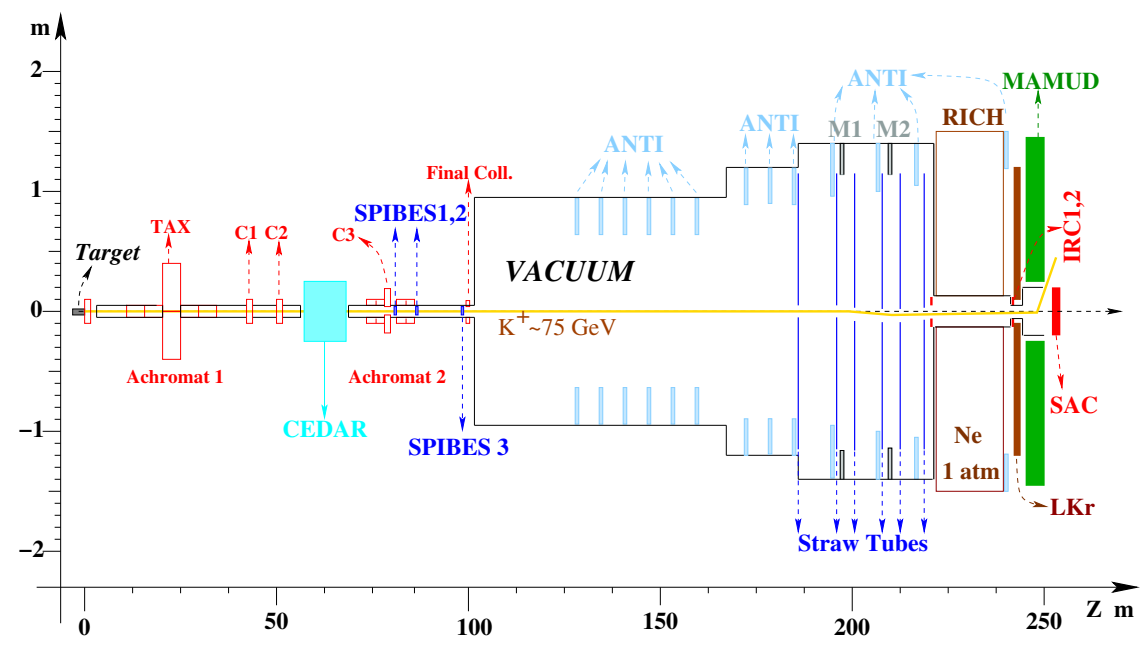

Figure 1: Layout of the experiment.

The experimental signature of a $K^{+} \rightarrow \pi^{+} v \bar{v}$ is one reconstructed positive track in the downstream detector. A beam and a pion tracking detectors provide a precise reconstruction of the 
kinematics, since the squared missing mass allows a kinematical separation between the signal and more than $90 \%$ of the total background, as shown in figure 2. In particular two signal regions can be defined where the backgrounds from $K^{+} \rightarrow \pi^{+} \pi^{0}$ and $K^{+} \rightarrow \mu^{+} v_{\mu}$ enter only because of non-gaussian tails in the squared missing mass resolution. However, the kinematics alone cannot provide a $10^{13}$ background rejection. A system of calorimeters for photon vetoes, muon veto and a RICH for positron, pion and muon separation is designed to fulfill these needs. Moreover, the detector layout gives redundancy both in kinematics reconstruction and particle identification allowing the background estimation directly from data.
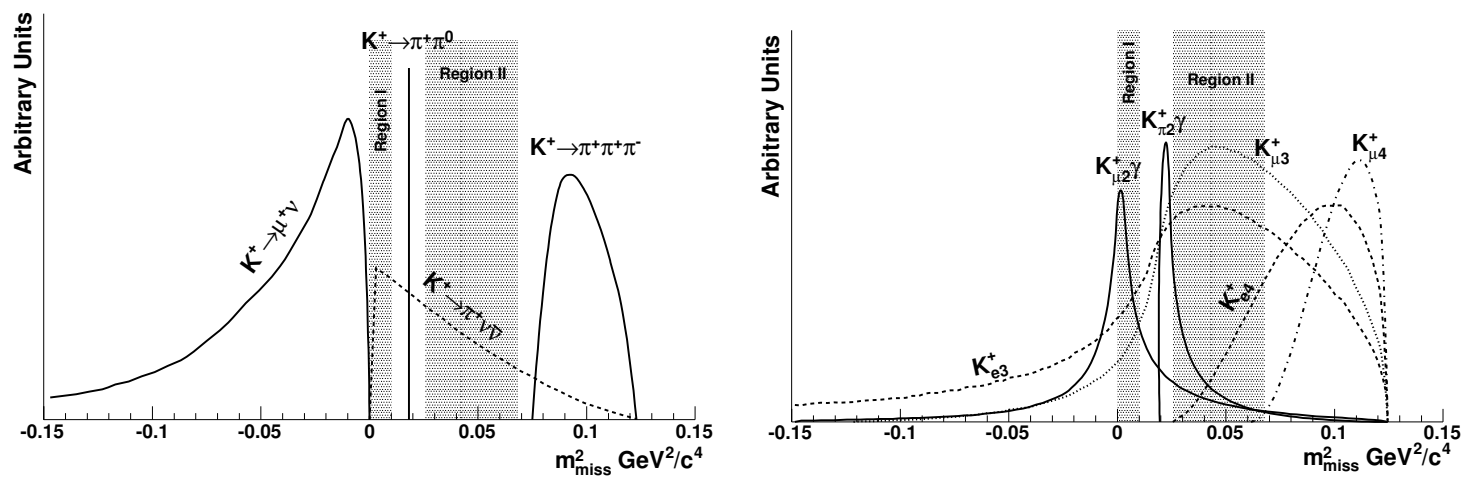

Figure 2: Squared missing mass for Kaon decays. The squared missing mass is defined as the square of the difference between the 4-momentum of the kaon and of the decayed track in the hypothesis that it is a pion.

\subsection{The beam line}

An intense $400 \mathrm{GeV} / \mathrm{c}$ proton beam, extracted from the SPS, produces a secondary charged beam by impinging on a Be target. A $100 \mathrm{~m}$ long beam line selects a $75 \mathrm{GeV} / \mathrm{c}$ momentum beam with $1.1 \%$ RMS momentum bite and an average rate of about $800 \mathrm{MHz}$ integrated over an area of $14 \mathrm{~cm}^{2}$. The beam is positron free and is composed by $6 \%$ of $K^{+}$. The average rate seen by the downstream detectors integrated on their surface is $\sim 11 \mathrm{MHz}$. This rate is due to kaon decays and accidentals coming from the beam line. The described beam line provides $5 \times 10^{12} \mathrm{~K}^{+}$decays, assuming $60 \mathrm{~m}$ decay region and 100 days of run at $60 \%$ of efficiency, which is a very realistic estimate based on the decennial NA48 experience at the SPS.

\subsection{The tracking system}

The beam tracker must be highly performing in terms of time and spatial resolution and able to sustain a particle rate of about $60 \mathrm{MHzcm}^{-2}$. The detector under study consists of three Si pixels stations (SPIBES) $60 \times 27 \mathrm{~mm}^{2}$, made up by $300 \times 300 \mu \mathrm{m}^{2}$ pixels each of them composed by a $200 \mu \mathrm{m}$ thick Si sensor and a chip $100 \mu \mathrm{m}$ thick, bump-bonded on the sensor. At least $200 \mathrm{ps}$ time resolution per station is required to provide a suitable tag of the kaon track. The design of a readout chip using a $0.13 \mu \mathrm{m}$ technology has been submitted in 2007. Radiation damage tests on sensor prototypes started in 2006 and are continuing in 2007.

The pion spectrometer is made by six straw chambers $0.5 \%$ radiation length thick placed directly in the same vacuum of the decay region to reduce the multiple scattering. Two magnets (M1 
and M2) divide pairs of chambers and provide redundant measurements of the particle momentum. Each station will operate at about $45 \mathrm{KHz}$ per tube on average, but, due to the beam halo, the region close to the hole will suffer up to $0.8 \mathrm{MHz}$ rate. The $\mathrm{R} \& \mathrm{D}$ program is started in 2006 and a reduced-size prototype will be tested in 2007 at CERN on the NA48/2 beam line

\subsection{The particle ID system}

Since the experiment uses an unseparated charged beam, the existing differential Cerenkov counter CEDAR [8], should provide the kaon identification. This detector is available at CERN, but upgrades are needed to adapt it to the new beam conditions. The R\&D is started with a test beam run in November 2006, mainly devoted to the study of the timing capability.

A $18 \mathrm{~m}$ long RICH located after the spectrometer and filled with $\mathrm{Ne}$ at atmospheric pressure is the core of the particle identification. A $11 \mathrm{~cm}$ radius beam pipe crosses the RICH and two tilted mirrors at the end reflect the Cerenkov light toward an array of about 2000 phototubes placed in the focal plane. This device is able to identify pions with momentum greater than $15 \mathrm{GeV} / \mathrm{c}$. Simulations showed that up to 40 photo electrons can be collected per track. Using phototubes of $1 \mathrm{~cm}$ diameter a better than $3 \sigma$ pion-muon separation for tracks with momentum below $35 \mathrm{GeV} / \mathrm{c}$ is achievable. The size of the phototubes is the main limitation to the Cerenkov angle resolution. The RICH must work also as a timing detector for the downstream track with a requested time resolution of $100 \mathrm{ps}$. The timing performances depend on the phototubes. To this purposes a set of phototubes were tested successfully with Cerenkov photons during the CEDAR test in November 2006 and using a laser beam. A full-length prototype will be integrated in the NA62 set-up and tested during the 2007 NA62 run at CERN.

\subsection{The Veto system}

A combination of calorimeters covering up to $50 \mathrm{mrad}$ serves to identify the photons produced in kaon decays.

Ring-shaped calorimeters (ANTI) cover the angular region between 10 and $50 \mathrm{mrad}$. They should guarantee the detection of photons down to $50 \mathrm{MeV}$ with $10^{-4}$ inefficiency at most and must be placed in vacuum. A scintillating fibers prototype has been built in Frascati in 2006. Tests on this device and on a Fermilab lead scintillator prototype have been carried on in 2007 using an electron beam at the BTF facility at LNF.

The existing NA48 liquid Kripton calorimeter (LKr) [9] covers the region between 1 and 10 mrad. A data analysis performed on $K^{+} \rightarrow \pi^{+} \pi^{0}$ decays collected by NA48/2 in 2004 shows that the inefficiency of the LKr is lower than $10^{-5}$ for photons with energy greater than $10 \mathrm{GeV}$. This result matches our requests in terms of efficiency for that range of energy. A run performed in October 2006 at the SPS using bremsstrahlung photons produced by electrons passing through the NA48 apparatus allows the LKr inefficiency between 2 and $10 \mathrm{GeV}$ to be also measured. The efficiency for photons below $2 \mathrm{GeV}$ is less critical. A program of consolidation and update of the readout electronics of the $\mathrm{LKr}$ is under way.

A set of calorimeters (IRCs, SAC) built with shashlyk technology cover the region below 1 mrad. Only photons with energy larger than $10 \mathrm{GeV} / \mathrm{c}$ illuminate this detector, making a $10^{-5}$ inefficiency achievable. A prototype was built and tested with electrons on the NA48 beam line in 2006. 
A $6 \mathrm{~m}$ long hadronic sampling calorimeter (MAMUD) provides a $10^{5}$ rejection of muons and deflect the charged beam out of the acceptance of the small angle calorimeter behind it.

\section{Performances}

A preliminary analysis has been done using Geant3 [10], Geant4 [11] and Fluka [12] based simulations. The total acceptance is about 17\%, showing that the target of $10 \%$ of signal acceptance is safely achievable even taking into account additional losses occurring in a real data taking. The use of the RICH constrains the accepted pion tracks within the $(15,35) \mathrm{GeV} / \mathrm{c}$ momentum range. The higher cut is an important loss of signal acceptance, but assures that events like $K^{+} \rightarrow \pi^{+} \pi^{0}$ deposit at least $40 \mathrm{GeV}$ of electromagnetic energy, making their rejection easier.

Many sources of background have been considered and just a simple counting of signal and background events in the signal regions indicates that the $10 \%$ background level is nearly achievable.

\section{Conclusions}

The ultra-rare $K \rightarrow \pi v v$ decay is a unique environment where to search for new physics. The NA48/3 experiment at CERN-SPS proposes to follow this road by collecting $O(100)$ events of the $K^{+} \rightarrow \pi^{+} v \bar{v}$ decay. Actually we are designing an experiment able to reach a $10^{-12}$ sensitivity per event employing existing infrastructures and detectors at CERN. The overall experimental design requires a sophisticated technology for which an intense $R \& D$ program has started.

\section{References}

[1] A. J. Buras, M. Gorbahn, U. Haisch and U. Nierste, JHEP 0611, 002 (2006) HEP-PH 0603079.

[2] W. M. Yao et al. [Particle Data Group], J. Phys. G 33 (2006) 1. JPHGB,G33,1.

[3] G. D’Ambrosio, G. F. Giudice, G. Isidori and A. Strumia, Nucl. Phys. B 645, 155 (2002) HEP-PH 0207036.

[4] G. Isidori, F. Mescia, P. Paradisi, C. Smith and S. Trine, JHEP 0608, 064 (2006) HEP-PH 0604074.

[5] M. Blanke, A. J. Buras, A. Poschenrieder, S. Recksiegel, C. Tarantino, S. Uhlig and A. Weiler, HEP-PH 0610298. JHEP 0701 (2007) 066.

[6] V. V. Anisimovsky et al. [E949 Collaboration], Phys. Rev. Lett. 93, 031801 (2004) HEP-EX 0403036.

[7] G. Anelli et al., CERN-SPSC-2005-013, SPSC-P-326.

[8] G. Bovet et al., CERN Report: CERN 82-12(1982).

[9] G. Unal, NA48 Collaboration, in: IX International Conference on Calorimetry, October 2000, Annecy, France, HEP-EX 0012011.

[10] CERN Program Library Long Writeup, W5013 (1993).

[11] J. Allison et al., IEEE Trans. Nucl. Sci. 53, 270 (2006). IETNA,53,270.

[12] A. Ferrari, P. R. Sala, A. Fasso and J. Ranft, CERN-2005-010. 